\title{
Corela
}

Cognition, représentation, langage

HS-7 | 2010

Espace, Préposition, Cognition

\section{Places-repère, localisation et routines : lorsque l'analyse du nom place rejoint celle de la préposition à}

\author{
Michel Aurnague
}

\section{OpenEdition}

Journals

Édition électronique

URL : http://journals.openedition.org/corela/919

DOI : 10.4000/corela.919

ISSN : 1638-573X

Éditeur

Cercle linguistique du Centre et de l'Ouest - CerLICO

Référence électronique

Michel Aurnague, « Places-repère, localisation et routines : lorsque l'analyse du nom place rejoint celle de la préposition à », Corela [En ligne], HS-7 | 2010, mis en ligne le 31 mai 2010, consulté le 20 avril 2019. URL : http://journals.openedition.org/corela/919; DOI : 10.4000/corela.919

Ce document a été généré automatiquement le 20 avril 2019.

\section{(c) (i) (2)(2)}

Corela - cognition, représentation, langage est mis à disposition selon les termes de la licence Creative Commons Attribution - Pas d'Utilisation Commerciale - Partage dans les Mêmes Conditions 4.0 International. 


\title{
Places-repère, localisation et routines : lorsque l'analyse du nom place rejoint celle de la préposition à
}

\author{
Michel Aurnague
}

\section{Introduction : de Vandeloise (1986) à Vandeloise (2001), des prépositions spatiales à l'ontologie des entités ${ }^{1}$}

1 L'examen des travaux de C. Vandeloise depuis le début des années 1980 jusqu'en 2007 (voir bibliographie complète dans ce numéro) fait apparaître deux périodes et deux types de questionnements autour desquels s'organisent les recherches de l'auteur. De 1980 à 1994, C. Vandeloise s'est essentiellement consacré à l'étude des prépositions spatiales du français et à la mise en place d'un cadre théorique à l'intérieur duquel le fonctionnement sémantique de ces marqueurs peut être décrit. La contribution la plus marquante de cette période est, sans nul doute, l'ouvrage L'espace en français: sémantique des prépositions spatiales paru en 1986. A partir de 1995, une nouvelle préoccupation se fait jour dans les recherches de C. Vandeloise, qui guidera peu ou prou les études menées jusqu'en 2007 : il s'agit de définir plus précisément les interactions entre l'«espace» (vide ${ }^{2}$ ) et la matière et, partant, de déterminer les propriétés ontologiques des entités et des concepts qui soustendent les descriptions spatiales. Dans le cadre de cette problématique, C. Vandeloise se penchera longuement sur les écrits d'Aristote (tout particulièrement sur la Physique) et publiera en 2001 Aristote et le lexique de l'espace: rencontres entre la physique grecque et la linguistique cognitive, livre emblématique de ce deuxième ensemble d'études.

Ces deux versants de l'œuvre de C. Vandeloise sont loin d'être disjoints et des préoccupations récurrentes parcourent les diverses publications portant, par exemple, sur les comparaisons/différences interlinguistiques ou sur l'acquisition des marqueurs spatiaux par les enfants (Aurnague 2008). Plus généralement, on constate que les deux périodes mentionnées envisagent le même objet d'étude - l'expression de l'espace dans la 
langue - sous deux perspectives différentes et complémentaires : celle des relations ou prépositions et celle de l'ontologie, davantage centrée sur les entités spatiales (que sur les relations). C'est cette complémentarité que nous tentons de mettre en évidence en prenant pour point de départ l'analyse de la préposition à publiée par C. Vandeloise en 1988 et celle qu'il proposera plus tard pour le nom générique de localisation place (Vandeloise 1999, 2001).

3 L'étude des emplois statiques de à (Vandeloise 1988) s'appuie sur deux règles d'usage censées cerner le fonctionnement de cette relation spatiale. La première règle s'applique aux cas où le site - entité de référence ou localisatrice - introduit par l'objet nominal de la préposition « localise » à proprement parler la cible - entité localisée -. La position du site doit alors être connue/spécifiée dans le savoir partagé des locuteurs et celui-ci remplit une « fonction de localisation ». Il n'est, dès lors, pas surprenant que les sites mis en jeu par ce type d'emploi soient identifiés par des noms propres (1) ou apparaissent à la suite d'un article défini (2) plutôt qu'en combinaison avec un article indéfini (3). La seconde règle d'usage proposée par C. Vandeloise regroupe les emplois statiques de à dans lesquels la cible participe à une « routine sociale » ou activité évoquée par le site (« site intégré »; 4-5). Bien que le contexte situationnel permette parfois d'identifier le site concerné, cet usage de à fait massivement appel à l'interprétation générique de l'article défini (cf. Tous les villageois sont à la fenêtre; Tous les vacanciers sont à la montagne).

(1) Léopold est à Liège

(2) Max est au hangar

(3) ??Léopold est à une maison

(4) Léopold est à la fenêtre

(5) Max est à la montagne

Nous avons montré, dans divers travaux (Aurnague 1996, 2004, Aurnague et al. 2005), que les sites correspondant au premier emploi de à (emploi statique localisateur) possèdent des propriétés ontologiques précises. On est ainsi en présence d'entités matérielles fixes/ stables dans un cadre de référence donné (fix) qui, par ailleurs, définissent/déterminent des « portions d'espace » (esp ; la notion de portion d'espace est développée dans la suite du texte ainsi que dans les notes 2 et 4 ). Les entités répondant à ces critères (+fix, +esp) sont qualifiées de "lieux» et elles englobent, de fait, les lieux géographiques puisque ceux-ci sont stables au sein du cadre de référence terrestre et présentent une portion d'espace adjacente à leur surface au sol, dans laquelle d'autres éléments peuvent être localisés (Vieu 1991). La contrainte indépendante de spécification de la position (spc) mise en évidence par C. Vandeloise joue un rôle important dans le fonctionnement de cette première règle d'usage et l'on est donc confronté à des « lieux spécifiés »: parce que les lieux géographiques identifiés par des noms propres (1) répondent à la fois à la définition de lieu mentionnée ci-dessus (+fix, +esp) et à la contrainte de spécification $(+\mathrm{spc})$, ils constituent des sites idéaux pour l'emploi localisateur de à. Les noms communs de lieux géographiques (+fix, +esp) se prêtent également à cet emploi à condition, toutefois, qu'ils soient introduits par un article défini (interprétation définie) qui garantisse un certain niveau de spécification (+spc; comparer (2) et (3)). Les entités violant l'un et/ou l'autre des critères caractéristiques des lieux sont, dans cette approche, considérées comme des « objets » et elles ne peuvent être sélectionnées par à dans son usage localisateur, quand bien même la position de ce site serait connue/spécifiée (article défini) : *Léopold est au rocher (+fix, -esp, +spc) ; ${ }^{*}$ La mouche est au verre (-fix, +esp, +spc). L'opposition objet vs. lieu ainsi posée peut être rapprochée de la distinction «participant» vs. «setting» de R. 
Langacker (voir contribution dans ce numéro), tout au moins pour ce qui concerne les représentants les plus canoniques de ces deux dernières catégories.

Quelques précisions doivent être apportées à ces hypothèses ontologiques. Indiquons, tout d'abord, qu'il ne s'agit pas d'une classification rigide et définitive (extérieure à la langue) et qu'une entité relevant d'une classe donnée (ex : objet) peut, dans un contexte situationnel particulier, acquérir des traits qui lui confèrent un statut distinct (ex : lieu) ${ }^{3}$. Deuxièmement, la définition de lieu telle qu'énoncée plus haut ne se limite pas aux seuls lieux géographiques et s'applique également aux Noms de Localisation Interne (NLI : haut, derrière, intérieur, bord, extrémité) : ces derniers identifient, en effet, des parties/zones stables au sein d'une entité-tout (+fix) auxquelles des portions d'espace sont fréquemment associées (+esp). Par ailleurs, le contenu sémantique de ces marqueurs précise - au moyen d'informations orientationnelles, topologiques ou liées à la distance la localisation des entités désignées $(+\mathrm{spc})$ et l'on a donc affaire à des lieux spécifiés pouvant, ici encore, se prêter au premier emploi de à : L'oiseau est au pied/centre/sommet de l'arbre $(+\mathrm{fix},+\mathrm{esp},+\mathrm{spc})^{4}$. Ajoutons, enfin, que les recherches sur les "génitifs » locatif et possessif du basque (Asher 2007, Aurnague 1998, 2002, 2004) ont mis en évidence le caractère ambivalent des habitations qui, tout en étant des lieux (fixité dans le cadre de référence terrestre, détermination d'une portion d'espace), possèdent des propriétés qui les rapprochent des objets (ex : structuration en « composants » ou parties fonctionnelles bien identifiées). Le terme « entité mixte » rend compte de ce statut particulier.

Les deux premières sections de cet article envisagent le fonctionnement du nom place lorsque celui-ci introduit des "places-repère ». Nous montrons que cet emploi peut être rapproché des deux règles (localisation et routines) dégagées par $\mathrm{C}$. Vandeloise pour analyser les usages statiques de la préposition à. Après une brève comparaison des " places-repère » et des « places-extension » (section 4), nous concluons en examinant les dépendances sémantiques que font apparaitre les noms génériques de localisation place et endroit et les NLI vis-à-vis de l'entité-cible et du cadre de référence ou entité-tout d'une description spatiale.

\section{Les places-repère en tant que lieux spécifiés}

7 Dans son analyse du mot place, C. Vandeloise $(1999,2001)$ propose de distinguer deux emplois principaux de ce terme, l'un qualifié de " place-extension » et l'autre de "placerepère ». Les emplois de type "place-extension " peuvent se référer à la " place-extension disponible » (qui consiste, selon l'auteur, en une « portion d'espace vide» : 6) ou bien à la "place-extension de l'objet » (dépendant de sa taille et de sa forme : 7):

(6) Il y a de la place dans l'armoire

(7) L'armoire prend beaucoup de place

8 Nous reviendrons, plus loin (section 4), sur les "places-extension" qui, comme l'illustrent les exemples ci-dessus, se singularisent par l'aspect massique ${ }^{5}$ des entités dénotées.

La notion de "place-repère " d'une entité mobile est définie par C. Vandeloise comme étant une portion d'espace ("entité spatiale») que détermine l'entité matérielle à laquelle la première (l'entité mobile) est associée dans un ordre établi. L'auteur différencie les «places-support » (la portion d'espace coïncide avec leur région d'« usage 
normal »: 8) des «places-contenant» (la portion d'espace correspond à leur « intérieur creux $»: 9)$ :

(8) Le piédestal est la place de la statue

(9) L'armoire est la place de la tasse

Nous avons noté dans (Aurnague 2004) que les places-repère, telles qu'introduites par C. Vandeloise, nécessitent la présence d'un cadre de référence à l'intérieur duquel ces éléments, dont on précisera plus loin la nature, sont positionnés et permettent la localisation d'autres entités: dans les exemples (8-9), il s'agit sans doute d'un jardin/ parc/square ou d'une pièce/habitation. Alors que les entités localisées sont généralement mobiles $^{6}$, la place-repère qui leur est assignée dans le cadre de référence (place-support dans (8) et place-contenant dans (9)) est, en revanche, fixe ou stable. Ajoutons que cette place-repère présente souvent un caractère connu/spécifié dans la connaissance partagée des locuteurs (cf. articles définis de (8-9)). Ces divers éléments - cadre de référence, position fixe/stable et spécification - découlent, en grande partie, du concept d'« ordre établi » utilisé par C. Vandeloise et jouent un rôle essentiel dans le caractère localisateur des places-repère, ou du moins de certaines d'entre elles. Nous approfondissons dans la suite plusieurs de ces points.

11 Une observation fine révèle que les places-repère sont, la plupart du temps, des portions d'espace pouvant être occupées par des cibles, ce que reflète d'ailleurs l'analyse de C. Vandeloise. Il ne faut cependant pas perdre de vue que, dans les descriptions spatiales comme, plus généralement, d'un point de vue cognitif -, les portions d'espace n'existent pas de manière véritablement autonome et sont dépendantes d'entités matérielles qui permettent de les déterminer/définir (ex : l'intérieur du verre, le dessous de la table, l'espace entre la table et l'armoire; la définition des "places-repère " proposée par $\mathrm{C}$. Vandeloise rend compte de cette dépendance). Il n'est, dès lors, pas surprenant que les places-repère soient identifiées par rapport à des éléments matériels, et ceci en recourant à des prépositions topologiques/internes (ex: sur, dans, à, locutions formées à partir de à et de $\mathrm{NLI})$ comme à des prépositions projectives/externes (ex : devant, près de) :

(10) La place de la table est dans le séjour/au centre du séjour

(11) La place de la chaise est près de l'armoire (du séjour)

La place-repère affectée à une entité-cible peut être "englobante» au sens où son extension dépasse celle de la cible ou, plus précisément, celle du « référent spatial » de la cible ${ }^{7}$. Certaines places-contenant proposées par C. Vandeloise illustrent cette possibilité, l'intérieur de l'entité jouant, dans son ensemble, le rôle de place-repère pour la cible à laquelle cette place est attribuée ((9) mais également La place de la tasse est (dans) l'armoire). Mais la référence aux entités matérielles permet, par raffinements successifs, de situer de plus en plus étroitement la portion d'espace correspondant à une place-repère (Aurnague 2004), si bien que celle-ci peut pratiquement correspondre au référent spatial de l'entitécible associée :

(12) Le livre est à sa place(,) dans l'armoire (du séjour), plus précisément sur l'étagère du haut, à droite, entre le vase et le coffret de disques...

Certaines constructions négatives reflètent d'ailleurs la précision avec laquelle une placerepère peut être amenée à localiser une entité-cible :

(13) Le vase est sur l'étagère mais pas exactement/vraiment à sa place

(14) Le vase n'est pas exactement/vraiment à sa place sur l'étagère

L'ambiguïté de (14) - imputable à la portée de la négation et à la fonction syntaxique du groupe prépositionnel sur l'étagère - met bien en évidence les variations possibles dans la 
détermination d'une place-repère : celle-ci peut, en effet, correspondre à l'ensemble de l'étagère (toutes configurations de type support confondues : place-support) ou bien se limiter à une zone particulière de cette étagère qui est alors amenée à fonctionner comme un cadre de référence (dans cette interprétation, (14) est équivalente à (13)).

Au-delà de son caractère un peu caricatural, (12) est un bon indice du degré de précision qu'il est possible d'atteindre en mettant à contribution des cadres de référence successivement enchâssés ainsi que certaines entités présentes dans ces cadres de référence ${ }^{8}$. Le choix de ces éléments est une question que nous n'approfondirons pas ici car il relève, pour l'essentiel, de contraintes et principes généraux régissant les descriptions spatiales 9 . Des restrictions particulières s'appliquent cependant aux cadres de référence et entités utilisés pour déterminer/situer les places-repère: les relations spatiales qui s'établissent entre eux semblent, en effet, devoir faire preuve d'une certaine stabilité. Ainsi, dans un contexte où le cartable de la phrase (15) se trouve localisé de façon provisoire ou (très) discontinue dans l'entrée, l'énoncé (16) revêtira certainement un caractère étrange. On retrouve, en réalité, au niveau de l'enchâssement des cadres de référence, la condition notée plus haut relative à la stabilité/fixité d'une place-repère à l'intérieur d'un cadre.

(15) La calculatrice est à sa place(,) dans le cartable

(16) ??\#La calculatrice est à sa place(,) dans l'entrée

Les stratégies de raffinements successifs recourant à des groupes prépositionnels spatiaux pour identifier «au plus près » les portions d'espace qui constituent les placesrepère ont pour pendant, du côté des entités, le fait que certains contenants ou supports peuvent « matérialiser » une place. Ce type de configuration, déjà perceptible dans (8), est illustré par les exemples suivants de C. Vandeloise (1999) basés, eux aussi, sur des placessupport :

(17) Ce fauteuil est la place du président

(18) Laurel a réservé une place à l'amphithéâtre

(19) Hardy a une place à l'avant du train

Dans d'autres cas, cette matérialisation prend la forme de places-contenant :

(20) Le disque compact est à sa place(,) dans la colonne (de CD)

(21) La bouteille est à sa place(,) dans le casier (à bouteilles)

Comme le suggèrent les énoncés (18-21), il n'est pas rare que les places matérialisées forment un groupe et s'organisent à l'intérieur du cadre de référence dans lequel elles se trouvent.

Les remarques effectuées jusqu'à ce point font apparaître que les places-repère sont généralement constituées par des portions d'espace (dans lesquelles il est possible de localiser des cibles) occupant une position stable dans un cadre de référence englobant: elles répondent donc, pour l'essentiel, à la notion de lieu que nous avons été amené à proposer (Aurnague 1996, 1998, 2004, Aurnague et al. 2005). Mais les portions d'espace étant déterminées par des entités matérielles, il est nécessaire, pour les identifier, de les mettre en relation avec celles-ci - au moyen, en particulier, de groupes prépositionnels localisateurs - ou de les ancrer matériellement à elles (places matérialisées). Dans le premier cas au moins (identification par des expressions spatiales), les places-repère assignées à des cibles sont introduites à travers la spécification de leur position et l'on est donc en présence de «lieux spécifiés ». Il n'est, du coup, pas surprenant que l'acception du terme place mettant en jeu le concept de place-repère soit étroitement associée à la 
préposition à (dont on a vu que la première règle d'usage sélectionne précisément des lieux spécifiés ; cf. (12-15) et (20-21)).

Les places matérialisées - parce qu'elles existent indépendamment des cibles qu'elles localisent - ont un fonctionnement un peu différent du point de vue de la spécification. Ainsi, la possibilité, pointée auparavant, qu'un ensemble de places s'organisent au sein d'un site/cadre de référence ouvre la voie à l'identification de ces places matérielles au moyen d'étiquettes ou de désignateurs rigides. Ces derniers sont un excellent moyen de spécifier la position des places-repère concernées et la combinaison à la préposition à en découle naturellement (22). Par ailleurs, si l'association du mot place et d'un article indéfini est plutôt rare (en particulier à l'intérieur d'un groupe prépositionnel en à :cf. section 1), elle apparaît sensiblement plus acceptable lorsqu'elle réfère à une place matérialisée incluse dans un ensemble d'entités de même nature $(23)^{10}$ que lorsqu'elle dénote une place-repère non matérielle (24). Ce type de construction - sous-tendu par ce que nous appelons la « spécification sous-spécifiée »- est discuté dans (Aurnague 2009).

(22) Max est (installé/assis) à la place $\mathrm{n}^{\circ} 40$ (dans/de la tribune)

(23) (?)Max est (installé/assis) à une place dans/de la tribune

(24) ??Le livre est à une place dans/de l'armoire

Il n'en reste pas moins que les places-repère sont, le plus souvent, des portions d'espace non matérialisées, identifiées à travers la spécification de leur position dans un cadre de référence. Ce procédé offre une grande facilité pour la création, en contexte, de «lieux spécifiés", comme l'illustrent les énoncés (25-26) prononcés dans le cadre d'une répétition, d'un tournage, etc. L'émergence des lieux spécifiés est ici naturelle et beaucoup plus souple que lorsqu'il s'agit, par exemple, de recatégoriser des objets au moyen d'un contexte approprié (ex : situations de jeu ; voir note 3$)^{11}$.

(25) A vos places!

(26) Chacun à sa place !

\section{Les places-repère en tant que routines sociales}

21 A côté des descriptions localisatrices analysées jusqu'ici, les places-repère entrent dans des constructions plus générales caractéristiques, entre autres, de certains vieux préceptes (Vandeloise 1999) :

(27) Un bar n'est pas la place d'une jeune fille

Comme le révèle le recours à l'article indéfini à valeur générique, (27) n'a pas pour but d'affecter une place-repère à une cible déterminée (à l'intérieur d'un cadre de référence donné). Ce genre d'énoncé fait appel à un ordre préétabli de nature « sociale » plutôt que spatiale et l'attribution d'une place-repère - ou, plus exactement, d'une catégorie de places-repère - à un type de cible se fonde sur des conventions qui peuvent être non seulement morales (27) mais, plus généralement, liées à l'utilisation et au comportement des entités qui nous entourent (28-29) :

(28) La place d'une tuile est sur un toit

(29) La place d'un livre est dans une bibliothèque

L'importance du lien conventionnel entre cible et site est ici fondamentale et son absence conduit d'ailleurs à des énoncés qui paraissent, pour le moins, étranges (30). La description localisatrice (31), parallèle à (30), ne pose, en revanche, aucun problème particulier, pour autant que les entités en présence soient contextuellement identifiables.

(30) \#La place d'une chaise est dans un garage 
(31) La place de la chaise est dans le garage

beaucoup plus rares ici, du fait, bien sûr, du caractère générique - non localisateur - des places-repère concernées. (40) et (41) indiquent cependant qu'elles ne sont pas tout à fait exclues. Cependant, et contrairement à (12), (15) et (20-21), la présence du groupe prépositionnel correspondant au site (ou, plus exactement, à la place-repère) est souvent nécessaire, la place-repère générique étant plus difficile à identifier dans le contexte ou le cotexte que la place-repère localisatrice. Inversement, l'ajout d'un groupe prépositionnel localisateur (ou d'une série de groupes prépositionnels) - très naturel dans le cas des places-repère «spatiales " - ôte à l'expression à sa place son interprétation générique ((40) : à l'université de Toulouse-Le Mirail ; (41) : au piano du fond de la salle $)^{14}$.

(40) Max est vraiment à sa place à l'université ! 
(41) Un pianiste est vraiment à sa place au piano !

En fin de compte, les places-repère décrites dans (Vandeloise 1999, 2001) se prêtent à des descriptions de nature assez différente. Dans un premier type d'emplois, ces placesrepère correspondent à des portions d'espace, matérialisées ou non, occupant une position fixe/stable dans un cadre de référence, position qui doit être, en outre, spécifiée ou connue : elles répondent alors aux critères définitoires des "lieux spécifiés". Les places-repère interviennent dans un second type d'emplois où leur rôle n'est pas précisément de localiser la cible : la mise en relation cible-site est ici réalisée sur la base de connaissances conventionnelles de nature morale, fonctionnelle, etc. En raison des contraintes strictes que font peser les routines sociales sur les couples cible-site, il n'est pas surprenant de trouver de nombreux sites intégrés dans ce deuxième usage des placesrepère. Bien qu'il ne soit pas absolu, un parallèle assez net paraît donc se dessiner entre ces deux emplois des places-repère et les règles d'usage proposées par $\mathrm{C}$. Vandeloise (1988) pour saisir le fonctionnement de la préposition à (emplois statiques), l'une sélectionnant des lieux spécifiés et l'autre faisant appel à des routines (sites intégrés). Il est, en conséquence, assez normal de voir le terme place se combiner presque exclusivement à cette préposition. Mais, par-delà les différences, une propriété commune réunit ces deux types de places-repère : elles sont, en effet, affectées, dans les deux cas, aux cibles des descriptions dans lesquelles elles apparaissent, que ce soit sur la base d'un ordre préétabli de type «spatial » ou d'un ordre conventionnel ayant un fondement plus "social ». Cette attribution des places-repère à des cibles se manifeste, sur un plan linguistique, par le recours à l'adjectif possessif.

\section{Places-repère vs. places-extension}

31 Nous revenons maintenant sur l'emploi de place qualifié par C. Vandeloise $(1999,2001)$ de «place-extension» (6-7). Cet emploi, nous l'avons déjà souligné, se caractérise par le comportement massique (non comptable) du terme place que met, tout particulièrement, en évidence l'usage de déterminants partitifs (de la place). Les places-repères, tout du moins spatiales/localisatrices, se comportent bien différemment car elles peuvent être «individualisées". Pour celles d'entre elles qui sont matérialisées, le recours à des déterminants numéraux est d'ailleurs systématiquement possible. Mais places-repère et places-extension présentent aussi certaines similitudes qui peuvent être mises au jour en se focalisant sur la notion de " place-extension de l'objet ».

La place-extension de l'objet ou " place-extension occupée » - par opposition à la " placeextension disponible »; cf. (Vandeloise 2001) - ne dépend pas de la position d'une entité à un moment donné mais plutôt de sa taille et de sa forme. C. Vandeloise $(1999,2001)$ précise que, dans le cas d'une entité pleine, la place-extension de cette entité correspond à son "intérieur plein » ainsi qu'à sa frontière. Pour une entité contenante, la placeextension ne se limitera pas à l'étendue de sa matière mais devra, dans de nombreux cas, également inclure l'extension de son "intérieur creux " ${ }^{15}$. Dans l'approche formelle de l'espace dans la langue que nous avons proposée avec L. Vieu (Aurnague \& Vieu 1993, Vieu 1991), une distinction assez nette est effectuée entre, d'une part, les propriétés géométriques liées au référent spatial d'une entité (cf. note 7) et, d'autre part, les propriétés fonctionnelles attribuées à l'entité elle-même. Dans le cadre de cette approche, la place-extension d'une entité spatiale relève, pour l'essentiel, du niveau de représentation géométrique : elle est conditionnée par la taille et la forme du référent 
spatial que définit l'entité (extension spatiale de sa matière) et, le cas échéant, son intérieur contenant ${ }^{16}$.

La place-extension étant une propriété des entités - basée sur leur taille et leur forme (ou, plus exactement, sur celles de leur référent spatial) -, on pourrait s'attendre à ce que cette dépendance se manifeste linguistiquement, en particulier par l'usage de l'adjectif possessif. Mais l'on a vu plus haut que le groupe nominal sa place est massivement utilisé pour dénoter la place-repère attribuée à une entité-cible. Cette tendance très forte est, selon nous, pour beaucoup dans l'impossibilité d'employer cette expression en relation avec la place-extension d'une entité (42). On remarquera, à ce propos, que la détermination possessive du terme volume - apparenté à place dans son emploi de type place-extension d'un objet (Vandeloise 1999) - est beaucoup moins problématique.

(42) Ce meuble est très lourd bien que *sa place/son volume soit limité(e)

La dépendance de la place-extension vis-à-vis de l'entité qu'elle caractérise constitue donc une propriété référentielle et cognitive significative qui rapproche, d'une certaine manière, cet usage de place de son emploi de type place-repère. Il a été indiqué, en effet, que les places-repère sont affectées/attribuées à des entités, ce que souligne élégamment l'expression «Une place pour chaque chose et chaque chose à sa place " (Vandeloise 1999). Cette formule suggère même la possibilité que s'établisse une relation biunivoque entre les éléments considérés : comme on a pu l'entrevoir avec les places-repère non matérialisées, il n'est, en particulier, pas rare qu'une cible coïncide avec la place-repère dans laquelle elle se trouve localisée (et contribue, ce faisant, à son identification). Placesrepère et places-extension partagent en tout cas une propriété commune en ce sens qu'elles manifestent, toutes deux, une forme de dépendance vis-à-vis de l'entité (cible) à laquelle elles sont associées. D'où le caractère fortement relationnel par rapport à la cible du terme place.

\section{Conclusions et perspectives : entité-cible, cadre de référence et dépendances}

35 L'observation des emplois de place basés sur la notion de place-repère (Vandeloise 1999, 2001) a révélé que ce terme figure dans des descriptions qui peuvent, pour une large part, être mises en parallèle avec les deux usages de la préposition à (configurations statiques : localisation et routines) dégagés dans (Vandeloise 1988). Alors que certaines placesrepère sont sous-tendues par un ordre préétabli de type spatial et identifient des lieux spécifiés (section 2), d'autres places-repère paraissent mettre en jeu un ordre plus social et conventionnel dont les routines constituent un exemple des plus appropriés (section 3). Cette heureuse convergence entre études menées à plusieurs années d'intervalle nous semble d'autant plus intéressante qu'elle n'a pas été voulue par l'auteur qui ne met, d'ailleurs, jamais en regard ces deux séries de résultats. Elle n'est pas pour autant fortuite et révèle la complémentarité des deux démarches adoptées par C. Vandeloise pour aborder l'espace dans la langue : étude des relations/prépositions locatives d'une part et mise en évidence des catégories ontologiques sur lesquelles s'appuient les descriptions spatiales d'autre part (ex : rapports entre " espace » et matière), ce dernier volet étant plus naturellement tourné vers l'examen des entités et des noms. Nous avons nous-même noté à plusieurs reprises l'utilité de ce double point de vue sur l'objet d'étude que constitue l'espace linguistique (Aurnague 2002, 2004). 

le sémantisme du nom place (dans son acception de type place-repère) et celui de la préposition à - et d'expliquer, par là, la cooccurrence fréquente de ces deux éléments -, les remarques relatives aux places-extension (section 4) nous ont conduit à adopter une perspective plus générale et à montrer que le terme place pris dans son ensemble est fortement relationnel vis-à-vis des cibles et entités auxquelles il s'applique. Afin de conclure ce travail, nous élargissons le propos aux NLI et au nom générique de localisation endroit que nous tentons de comparer à place sur la base, précisément, des dépendances sémantiques que ces termes dessinent par rapport à la cible et au cadre de référence ou entité-tout. Pour ce qui est du nom place, cette comparaison se limite essentiellement aux places-repère non matérielles.

Les places-repère étant affectées à des cibles qu'elles servent à localiser, il en résulte, comme on l'a noté, que le nom place est sémantiquement dépendant de ces entités (43b-c). La création des places-repère est d'autant plus aisée que celles-ci sont très souvent non matérielles et peuvent être identifiées par raffinements successifs au sein d'un cadre de référence (section 2). Mais cette immatérialité semble ici aller de pair avec le fait que le cadre de référence ne peut être constitué d'un ensemble d'éléments (" places ») de même nature. Ces deux propriétés - immatérialité des places-repère et absence de structure collective du cadre de référence - sont certainement à l'origine de la difficulté à décrire la relation entre une cible et un cadre englobant au moyen de constructions de la forme une place de Dét Ncdr ou la place... de Dét Ncdr (44a-b ; voir aussi (24)). Ainsi qu'il a été relevé à la section 2 , la nature matérielle de certaines places-repère et la possibilité que le cadre de référence soit alors composé d'éléments du même type rendent les constructions considérées nettement plus acceptables (ex : une place de la tribune/du wagon; la place la plus confortable de la tribune/du wagon; cf. (22-23)).

(43) (a) la place où se trouve le vase (b) la place du vase (c) sa place

(44) (a) ??une place de l'armoire (b) ?(?)la place la plus visible de l'armoire

Dans son usage «dépendant", le nom générique de localisation endroit permet d'identifier une zone à n'importe quelle(s) position(s) d'une entité-tout servant de cadre de référence (Aurnague 2009). Quand cette identification se fonde sur des propriétés liées au tout - bien qu'accidentelles ou contingentes -, endroit fait preuve d'une certaine relationnalité par rapport à la cible/propriété concernée (45b). Les descriptions "génitives » du type (45b) s'avèrent toutefois exclues lorsque la désignation d'un « endroit » fait appel au procédé plus externe de localisation d'une cible (ex : l'endroit où se trouve l'abeille; *l'endroit de l'abeille; *son endroit) ${ }^{17}$. D'autre part, on a montré dans (Aurnague 2009) que le sémantisme d'endroit suppose la décomposition (au moins virtuelle) de l'entité-tout ou cadre de référence auquel ce terme est associé en un ensemble d'entités similaires. Cette particularité d'endroit - en lien direct avec sa nature dépendante/relationnelle - joue, selon nous, un rôle essentiel dans l'acceptabilité des expressions (46a-b) mettant en relation la zone identifiée et le cadre englobant.

(45) (a) l'endroit où se trouve la blessure (b) l'endroit de la blessure (c) *son endroit

(46) (a) un endroit de l'armoire (b) l'endroit le plus visible de l'armoire

Nous en venons enfin aux NLI dont on peut dire qu'ils découpent le cadre de référence constitué par une entité-tout en un ensemble de zones (Aurnague 1996, 2004, Borillo 1999). Le contenu sémantique de ces marqueurs spécifie la localisation des éléments introduits au moyen d'informations orientationnelles (ex: haut, avant, gauche), topologiques (intérieur, bord, coin) ou relevant de la distance (ex : centre milieu, extrémité). 
Loin d'être identifiées de manière isolée (distribution arbitraire), les zones ainsi découpées s'organisent en un système de localisations antagonistes définissant des oppositions graduelles : haut vs. bas, avant vs. arrière, gauche vs. droite, intérieur vs. extérieur-limite(s), centre-milieu vs. périphérie-extrémités. Le fonctionnement des NLI apparaît donc largement tourné vers le cadre de référence/tout et ses propriétés internes (orientationnelles, topologiques, etc.) et ces termes n'entretiennent pas de dépendance particulière par rapport aux cibles qu'ils sont amenés à localiser (47b-c, avec interprétation identique à $47 a)$. Une dépendance référentielle forte existe, en revanche, vis-à-vis du cadre de référence ou entité-tout et, lorsque les zones identifiées sont multiples (ex : angle, bord, coin, extrémité), des constructions du type (48a-b) sont possibles.

(47) (a) l'angle où se trouve la tache (b) ??*l'angle de la tache (c) *son angle

(48) (a) un angle de l'armoire (b) l'angle antérieur gauche de l'armoire

dépendance cadre de réf.

NLI

endroit

dépendance cible

place (non matériel)

Figure 1 sémantiques mises au jour. L'examen détaillé de noms génériques de localisation tels que place et endroit est, en particulier, nécessaire dans la perspective d'une telle tâche (Borillo 2006, Huyghe 2006a, 2006b, 2007). L'analyse de place et endroit abordés à travers leur association à la préposition à ainsi que les recherches sur les NLI apportent cependant des informations classificatoires et ontologiques qui ne sont pas étrangères au fonctionnement relationnel de ces éléments lexicaux. Ainsi, les places-repère immatérielles répondent aux contraintes des lieux spécifiés et la souplesse avec laquelle elles émergent et sont assignées à des cibles les rend assez similaires aux lieux spécifiés créés en contexte (section 2 et note 3). L'emploi dépendant d'endroit fait, lui, clairement référence à un cadre englobant mais la faculté d'identifier des zones à n'importe quelle position de l'entité-tout - au moyen de propriétés accidentelles/contingentes ou de la simple localisation d'une cible - s'accompagne d'un sémantisme non spécifié du point de vue de la localisation (on est en présence de lieux non spécifiés : Aurnague 2009). Les NLI, au contraire, indiquent précisément la localisation des zones qu'ils désignent à l'intérieur d'un cadre de référence/tout qu'ils contribuent à structurer : leur contenu sémantique, éminemment relationnel vis-à-vis de l'entité-tout, satisfait la définition de lieu spécifié et leur dimension localisatrice les rapproche, d'une certaine façon, des noms propres de lieux géographiques (Aurnague 1996, 2004). 


\section{BIBLIOGRAPHIE}

Asher, N. (2007). Objects, locations and complex types. In M. Aurnague, M. Hickmann \& L. Vieu (eds), The categorization of spatial entities in language and cognition. Amsterdam : John Benjamins (Human Cognitive Processing 20), pp. 317-361.

Aurnague, M. (1996). Les Noms de Localisation Interne : tentative de caractérisation sémantique à partir de données du basque et du français. Cahiers de Lexicologie, 69, pp. 159-192.

Aurnague, M. (1998). Basque genitives and part-whole relations: typical configurations and dependences. Carnets de Grammaire, 1. Toulouse : rapport ERSS.

Aurnague, M. (2002). Relations de partie à tout, configurations typiques et dépendances : analyse sémantique de quelques constructions génitives du basque. Revue de Sémantique et Pragmatique, 11, pp. 69-85.

Aurnague, M. (2004). Les structures de l'espace linguistique : regards croisés sur quelques constructions spatiales du basque et du français. Leuven/Paris : Peeters (Bibliothèque de l'Information Grammaticale 56).

Aurnague, M. (2008). Claude Vandeloise: obra baten mugarriak [Claude Vandeloise : les jalons d'une œuvre]. Gogoa, 8 (1), pp. 9-24.

Aurnague, M. (2009). A cet endroit vs. dans un tel endroit : ce que à nous dit d'endroit et vice-versa. Langages, 173 (Approches récentes de la préposition, D. Stosic \& W. De Mulder (eds)), pp. 34-53.

Aurnague, M., Hickmann, M., \& Vieu, L. (2005). Les entités spatiales dans la langue : étude descriptive, formelle et expérimentale de la catégorisation. In C. Thinus-Blanc \& J. Bullier (eds), Agir dans l'espace. Paris : Editions de la Maison des Sciences de l'Homme (Cognitique), pp. 217-232.

Aurnague, M. \& Vieu, L. (1993). A three-level approach to the semantics of space. In C. ZelinskyWibbelt (ed), The semantics of prepositions: from mental processing to natural language processing. Berlin : Mouton de Gruyter (Natural Language Processing 3), pp. 395-439.

Borillo, A. (1998). L'espace et son expression en français. Gap/Paris : Ophrys.

Borillo, A. (1999). Partition et localisation spatiale : les Noms de Localisation Interne. Langages, 136 (Sémantique lexicale et grammaticale, Y.Y. Mathieu (ed)), pp. 53-76.

Borillo, A. (2001). La détermination et la préposition de lieu à en français. In X. Blanco, P.A. Buvet \& Z. Gavriilidou (eds), Détermination et formalisation. Amsterdam : John Benjamins (Linguisticae Investigationes Supplementa 23), pp. 85-99.

Borillo, A. (2006). Y a-t-il un endroit qui, tout compte fait, ne soit pas un endroit du monde? In M. Riegel, C. Schnedecker, P. Swiggers \& I. Tamba (eds), Aux carrefours du sens. Hommages offerts à Georges Kleiber pour son $60^{e}$ anniversaire. Leuven/Paris : Peeters, pp. 471-485.

Herskovits, A. (1986). Language and spatial cognition: an interdisciplinary study of the prepositions in English. Cambridge : Cambridge University Press.

Huyghe, R. (2006a). Entre localisation et partition, le cas d'endroit. In G. Kleiber, C. Schnedecker \& A. Theissen (eds), La relation partie-tout. Leuven/Paris : Peeters (Bibliothèque de l'Information Grammaticale 59), pp. 41-57. 
Huyghe, R. (2006b). Les noms génériques d'espace en français. Thèse de Doctorat, Université de Lille 3.

Huyghe, R. (2007). Les noms de localisation spatiale face au problème de la synonymie. Cahiers du LRL, 1 (Interprétation : aspects sémantiques et pragmatiques. Entre théorie et applications, L. Fraczak \& F. Lebas (eds)), pp. 85-103.

Muller, P. (2007). The temporal essence of spatial objects. In M. Aurnague, M. Hickmann \& L. Vieu (eds), The categorization of spatial entities in language and cognition. Amsterdam : John Benjamins (Human Cognitive Processing 20), pp. 337-361.

Vandeloise, C. (1986). L'espace en français : sémantique des prépositions spatiales. Paris : Seuil (Travaux Linguistiques).

Vandeloise, C. (1987). La préposition à et le principe d'anticipation. Langue Française, 76, pp. 77-111.

Vandeloise, C. (1988). Les usages statiques de la préposition à. Cahiers de Lexicologie, 53, pp. 119-148.

Vandeloise, C. (1999). Une place pour chaque chose et chaque chose à sa place. In M. Plénat, M. Aurnague, A. Condamines, J.P. Maurel, C. Molinier \& C. Muller (eds), L'emprise du sens : structures linguistiques et interprétations, Mélanges offerts à Andrée Borillo. Amsterdam : Rodopi (Faux Titre 174), pp. 303-321.

Vandeloise, C. (2001). Aristote et le lexique de l'espace : rencontres entre la physique grecque et la linguistique cognitive. Stanford, CA : CSLI.

Vieu, L. (1991). Sémantique des relations spatiales et inférences spatio-temporelles : une contribution à l'étude des structures formelles de l'espace en langage naturel. Thèse de Doctorat, Université Paul Sabatier, Toulouse.

\section{NOTES}

1. Cet article est dédié à Claude Vandeloise (1944-2007) dont les recherches sur l'espace en français ont été et resteront pour moi un exemple de profondeur et de cohérence ainsi qu'une source d'inspiration toujours renouvelée.

2. Le nom " espace », comme l'adjectif «spatial» (dans " entité spatiale »), est souvent utilisé, dans les publications de cette deuxième période, en référence à l'espace vide ou inoccupé. Pour notre part, nous préférons introduire les entités immatérielles en utilisant, selon les cas, les concepts de portions/morceaux d'espace ou de référent spatial d'une entité.

3. Une entité ayant les caractéristiques d'un objet (absence de fixité et/ou de portion d'espace) peut, provisoirement et dans des conditions bien déterminées, être recatégorisée comme un lieu (Aurnague 2004). C'est le cas, entre autres, d'objets tels qu'une chaise ou un tabouret (-fix, -esp) utilisés comme repères dans une «situation de jeu " (ex: Maintenant, je suis à la chaise/au tabouret!). Ce type d'exemple suppose un contexte situationnel bien précis et, notamment, la présence d'un cadre de référence englobant au sein duquel les «objets » jouent un rôle de jalon et revêtent, très provisoirement, un caractère fixe/stable et spécifié (tout en déterminant, dans leur proximité, des portions d'espace localisatrices). Dans ces situations « construites ", comme dans le cas des NLI (voir plus loin), fixité et spécification de la position fonctionnent donc conjointement.

4. Cette phrase peut être mise en regard de la construction inacceptable *L'oiseau est à l'arbre. De même qu'un rocher ou un poteau (auxquels n'est associé aucun intérieur immatériel), un 
arbre est une entité fixe dans le cadre de référence terrestre (+fix) qui, tout au moins dans nos cultures, ne définit pas de portion d'espace durablement utilisée pour la localisation (-esp). Certaines formes de portions d'espace peuvent, le cas échéant, être attribuées à la ramure des arbres (intérieurs de type «contour»; Herskovits 1986, Vieu 1991) mais ces "intérieurs" ne servent pas à localiser, de façon habituelle, d'autres entités et présentent, en conséquence, un caractère beaucoup moins stable et saillant que les véritables intérieurs « contenants ».

5. Nous préférons le terme «massique » à celui de «massif» utilisé dans d'autres travaux. Les noms ou termes de masse introduisant des entités massiques/massives admettent, entre autres choses, des emplois partitifs (cf. section 4).

6. Un énoncé tel que L'église est à sa place n'est, en effet, acceptable que dans des conditions restreintes. C'est le cas, par exemple, lorsque la cible est, par essence, un élément mobile (c'est-àdire un « objet» : décor, morceau de puzzle, etc.) ou lorsque le contexte situationnel rend cette entité potentiellement muable/éphémère (L'église est encore à sa place !)

7. A la suite de (Vieu 1991), nous distinguons une entité de son référent spatial : celui-ci correspond à la portion d'espace que détermine cette entité (en l'occupant matériellement) et avec laquelle elle coïncide à un moment donné. Une telle distinction s'avère nécessaire si l'on veut rendre compte du fait que des entités différentes peuvent avoir le même référent spatial : la bague vs. l'or de la bague, l'intérieur du verre vs. l'eau contenue dans le verre. L'approche peut être élargie à l'espace-temps pour peu que l'on décide d'affecter aux entités des individus quadridimensionnels (trois dimensions spatiales et une dimension temporelle). Un tel choix ouvre la voie à un traitement homogène des entités spatiales et des entités temporelles (éventualités/procès) (Muller 2007).

8. Cet énoncé (12) fait appel à, au moins, trois cadres de référence imbriqués - le séjour, l'armoire et l'étagère - ainsi qu'à deux entités situées sur l'étagère - le vase et le coffret -.

9. Il s'agit ici, principalement, de conditions relatives au choix du site telles que l'unicité ou non d'une entité dans le contexte situationnel, la localisation du locuteur et de l'interlocuteur par rapport à elle (au moment de l'énonciation), le «principe du site minimal» (Vieu 1991) et, probablement, d'autres contraintes pragmatiques.

10. Le niveau d'acceptabilité de cette construction n'est pas équivalent pour toutes les entités matérialisées formant un ensemble (ex : ??Le disque compact de Rameau est à une place dans/de la colonne). Des restrictions supplémentaires s'appliquent certainement, parmi lesquelles la possibilité d'identifier l'élément matérialisé par le mot place - dans le cas de la colonne, on parlera plutôt de « compartiment »-, le type de configuration qui s'établit entre la cible et le site (support, contenance...), etc.

11. Fixité/stabilité dans un cadre de référence et spécification de la position sont étroitement liées dans le fonctionnement des places-repère non matérialisées : elles apparaissent, sur ce plan, relativement proches des NLI et, surtout, des lieux spécifiés créés en contexte. Ces deux propriétés sont plus indépendantes pour les places-repère matérialisées et l'on peut mettre en parallèle le comportement de ces entités et celui des lieux géographiques.

12. Notons que, tout comme le site, la cible peut se voir associée à un article défini : La place de la jeune fille n'est pas au bar; La place du prêtre est à l'église (cf. également (37)).

13. Ceci rejoint les observations effectuées dans (Borillo 2001) et (Aurnague 2004) selon lesquelles les emplois de type «routine » de à n'impliquent pas systématiquement une lecture générique de l'article introduisant le site et peuvent s'accommoder d'une certaine détermination de ce site. La ligne de partage entre les deux usages de à (localisation et routine) ne serait, de ce point de vue, pas aussi tranchée que le laissent supposer les travaux de C. Vandeloise (1987, 1988).

14. Dans le cas de (41), la lecture localisatrice de à sa place - consécutive à l'ajout du groupe prépositionnel - entre potentiellement en conflit avec le caractère générique du sujet (un 
pianiste) si bien que la phrase résultante peut (sauf contexte spécifique) paraître curieuse. Je remercie le relecteur qui a pointé ce phénomène.

15. C. Vandeloise souligne cependant que la prise en compte ou non de l'intérieur creux dans la place-extension d'une entité peut dépendre de facteurs structurels (contenant fermé ou en partie ouvert) aussi bien que partiellement «contextuels» (possibilité d'emboîter/empiler certaines entités les unes dans/sur les autres).

16. Deux entités dont les référents spatiaux sont de taille et de forme identiques auront, par conséquent, la même place-extension. Elles pourront systématiquement «tenir » dans les mêmes places-repère.

17. En dépit de son caractère partiellement relationnel par rapport à la cible, endroit se distingue de place en ce qu'il circonscrit des zones qui ne sont pas attribuées/affectées aux cibles ou propriétés en présence (d'où l'exclusion des expressions avec adjectif possessif). L'association stable ou régulière de la cible et de la zone identifiée est un facteur important qui paraît favoriser l'émergence de constructions apparentées à $(45 \mathrm{~b}-\mathrm{c})$ : l'endroit où se trouve/pose habituellement l'abeille; l'endroit préféré de l'abeille; son endroit préféré. Ajoutons que si des tours du type à son/mon endroit sont possibles avec des compléments animés, ils doivent être clairement distingués des constructions considérées ici (par leur forme comme par leur sens). Ces tours sont, en effet, basés sur une véritable locution prépositionnelle (à l'endroit de (quelqu'un)) dont le contenu sémantique est proche des expressions à l'égard de ou envers.

\section{RÉSUMÉS}

Ce travail revient sur les analyses proposées par C. Vandeloise pour rendre compte du fonctionnement statique de la préposition à ainsi que du nom générique de localisation place. Il est montré que si l'assignation d'une "place-repère " à une entité-cible (entité localisée) repose souvent sur la spécification de la position à l'intérieur d'un cadre de référence ( «lieu spécifié »), elle peut aussi faire appel à un ordre plus social ou conventionnel (non spatial), ces deux types de places-repère étant en relation directe avec les règles d'usage qui gouvernent les emplois statiques de à (localisation et routines sociales). La convergence de ces deux séries d'études illustre, de façon saisissante, la complémentarité de deux points de vue possibles sur l'expression de l'espace dans la langue: analyse des prépositions/relations d'une part et mise en évidence des propriétés ontologiques des entités spatiales d'autre part. La réflexion se prolonge à travers la comparaison des dépendances sémantiques que dessinent les noms génériques de localisation place et endroit ainsi que les Noms de Localisation Interne (NLI) vis-à-vis de l'entité-cible et du cadre de référence ou entité-tout d'une description.

This work addresses the analysis proposed by C. Vandeloise in order to capture the static functioning of the preposition à ('at'; also 'in' and 'on' in English) and the semantics of the generic localization noun place ('place'). It is shown that while the assignation of a "mark-place" to a target (localized entity) is often based on the specification of the position within a frame of 
reference ("specified location"), it can also involve a more social or conventional (non spatial) order. These two types of mark-places prove to be in direct relation with the usage rules governing the static uses of $\grave{a}$ (localization and social routines). The convergence of these two series of studies strikingly illustrates the complementary character of two possible points of view on the expression of space in language: analysis of prepositions/relations on the one hand and search for the ontological properties of spatial entities on the other hand. Reflection goes on with a comparison between the semantic dependencies displayed by both the generic localization nouns place and endroit and the Internal Localization Nouns (ILNs), with respect to the target entity and the frame of reference or whole entity of a description.

\section{INDEX}

Keywords : Internal Localization Nouns (ILNs), mark-place, reference of frame, relation, space, target, whole entity

Mots-clés : cadre de références, entité-cible, entité-tout, espace, Noms de Localisation Interne (NLI), place-repère, relation

\section{AUTEUR}

MICHEL AURNAGUE

CLLE-ERSS (UMR 5263), CNRS \& Université de Toulouse-Le Mirail 